\title{
SURVEY OF INDUSTRIAL APPLICATIONS OF CONDUCTING POLYMERS
}

\author{
S. RoTH \\ Max-Planck-Institut für Festkörperforschung \\ Heisenbergstr. 1, D-70569 Stuttgart, Germany \\ W. Graupner \\ Institut für Festkörperphysik, Technische Universität Graz \\ Petersgasse 16, 8010 Graz, Austria \\ AND P. MCNEILlis \\ Institut Charles Sadron, CNRS, 6 rue Boussingault, 67083 Strasbourg, France \\ Seventeen years after the first publication of doped polyacetylene we \\ have already seen the appearance and disappearance of commercial products \\ based on conducting polymers. In the present report we want to summarize \\ the status quo in this field paying attention to existing products, laboratory \\ demonstration set-ups and promising concepts.
}

PACS numbers: 72.80.Le, 81.90.+c, 89.20.+a

\section{Introduction}

A civilization often is named after the material which dominates in tool-making. Therefore historians speak of the Stone Age, Bronze Age, and Iron Age. Following these lines our present time would be called Silicon Age [1]. Without doubt, information technology has a decisive influence on all aspects of our everyday life and the material basis of information technology is silicon. Will there be a Gallium-Arsenide Age in the future? Or a Diamond Age, or an Organic Age, a Molecular Electronics Age? Diamond can be used as a semiconductor at higher temperatures than silicon, and diamond has a better thermal conductivity. Organics (phtalocyanines, conjugated polymers, dye-stuffs etc.) allow for a higher integration density than inorganic semiconductors. Will the emergence of diamond, polymers, and fullerenes as high-tech materials lead to a Carbon Age following the Silicon Age?

Mankind has invested hundreds of thousands of man-years into research and development (R\&D) of silicon technology and consequently silicon is the 
best-known solid today. Some people even claim that a silicon single crystal is the best-known section of the universe, exhibiting less imperfections than there are rest gas atoms in ultrahigh vacuum. Will we be able to replace silicon technology by something better? There is the sarcastic statement: "Gallium-arsenide was, is, and ever will be the semiconductor of the future." Would not this be even more true for organics and other exotic materials? There is a "technological nucleation problem" and if a new material is not much better, more than an order of magnitude better, it will not replace the conventional material.

What are conducting polymers? We should better speak of conjugated polymers, because these materials are not only interesting in their "doped" conducting state, but also undoped or weakly doped as semiconductors. The chemical structure of some of the most important conjugated polymers is shown in Fig. 1. Polyacetylene is the prototype for scientific investigations, for technical application

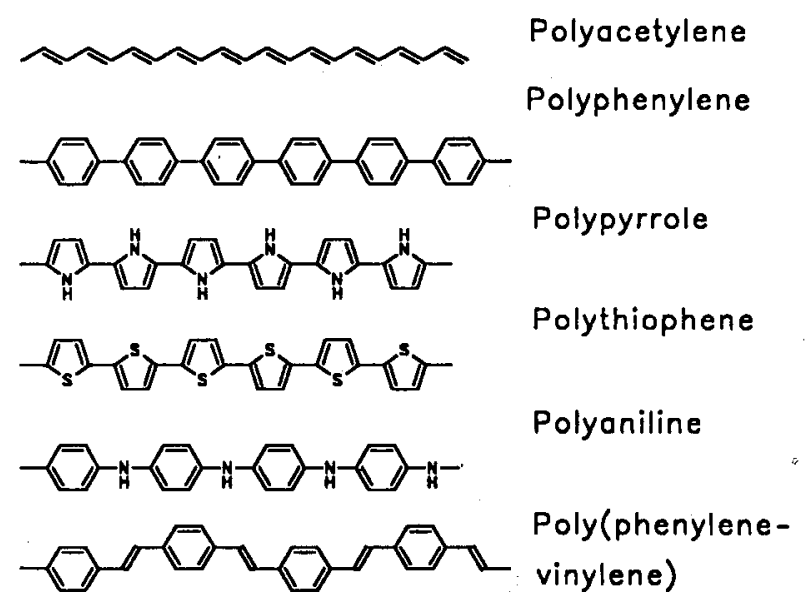

Fig. 1. Chemical structure of some of the most important conjugated polymers.

the other polymers are more important. Undoped conjugated polymers are semiconductors with band gaps ranging from 1 to several $\mathrm{eV}$, therefore their room temperature conductivity is very low, $10^{-8} \mathrm{~S} / \mathrm{cm}$ and lower. But by doping (chemical redox treatment) the conductivity can be changed by many orders of magnitude. The highest value reported so far has been obtained in iodine-doped polyacetylene $\left(>10^{5} \mathrm{~S} / \mathrm{cm}\right)$ and the predicted theoretical limit is about $2 \times 10^{7} \mathrm{~S} / \mathrm{cm}$, more than a magnitude higher than that of copper. The conductivity in the other conjugated polymers saturates at some $100 \mathrm{~S} / \mathrm{cm}$, but for many applications even $10^{-3} \mathrm{~S} / \mathrm{cm}$ is sufficient, e.g. for electrostatic dissipation [2].

Market predictions for new materials are always risky. It is generally agreed that in the near future organics will not replace silicon and conjugated polymers will not replace copper. But there will be enough economic niches where conjugated polymers can be commercialized. In a study of 1992 [3] predicting the market for polyaniline in 2000 , potential sales were estimated for US\$ 1 Billion world-wide, 
assuming a projected price of US $\$ 35 / \mathrm{lb}$. That is no technological revolution, but it will allow some companies to survive. Similarly, a study on organic nonlinear optical materials predicts a US market of $\$ 150.000$.- for the year 2000 [4], which upscaled would correspond to US\$ 0.5 Billion world-wide (conjugated polymers and conjugated oligomers are an important class of organic nonlinear optics (NLO) materials).

\section{Applications involving conductivity}

\subsection{Electrostatic dissipation: packing materials}

Modern electronic devices are easily damaged by electrostatic fields arising from friction. Therefore chips are usually carried in antistatic boxes and if packed in plastic foils ("blister belts") these foils must be sufficiently conducting in order to dissipate electrostatic charges. This can be achieved by using a compound of a conventional plastic with a doped conjugated polymer [5]. Alternatively, needles or dendrites of conducting organic charge transfer salts can be incorporated into the conventional polymer. Such packing foils are actually in use in electronics factories.

\subsection{Electrostatic dissipation: floor carpets}

Almost everybody of us has experienced the unpleasant feeling of an electric discharge when touching the door knob after walking over a synthetic wall-to-wall carpet on a dry winter day. To reduce this effect, antistatic additives are mixed into the carpet polymer. The conductivity of conventional antistatically treated plastics depends on the humidity of the air. A Finnish company now has announced commercialization of plastic floor tiles based on the conjugated polymer polythiophenes. The conductivity of these tiles is independent on humidity.

\subsection{Electrostatic dissipation: electron beam lithography}

Today integrated circuits are usually structurized by photolithographic techniques. In this process a pattern is written in an organic layer (photoresist). Then the pattern is transferred to the semiconductor by etching. Spatial resolution is limited by the wavelength of light. For further miniaturization, radiation with smaller wavelength has to be used: X-rays or electrons. Unlike light particles, however, electrons are electrically charged. Thus electrons trapped in the resist deflect the further electrons and limit the resolution, if they are not removed. Consequently a conducting resist is needed. This can be obtained by mixing metal particles into the resist - or by using particles of a conducting polymer. Presently, several $R \& D$ projects are devoted to electrodissipative resists for electron beam lithography [6].

\subsection{EM shielding}

Electromagnetic shielding and electromagnetic compatibility are important issues for electric and electronic appliances. For example, during take-off and landing of an aircraft lap-top computers have to be switched off in order not to interfere with the pilot's navigation system. When in the old days computers were in a metal 
cage this problem did not exist. Now the engineers try to make the plastic housing of electronic equipment conducting to avoid electromagnetic radiation from going in or out. A particular case are mobile telephones ("handies"), which emit so intensive microwave radiation that it even might be biologically hazardous. Shielding electromagnetic waves in the near field (i.e. close to the source) is complicated not only from the technical but also from the physical point of view [7]. Thermoplastic polymers with carbon-black filler, metal chips or steel needles are one solution, blends with polyanilines or polypyrrole particles another. In this context it is perhaps also worthwhile noting that during the Gulf War polypyrrole coated nets were used for radar camouflage of army tanks.

\subsection{Capacitors}

To have a large capacity a capacitor should have a large surface and a thin dielectric layer. Electrolyte capacitors use the large surface area of specially treated aluminium, the thin aluminium-oxide layer which automatically forms when aluminium metal is brought into contact with a liquid electrolyte, and the electrolyte as second capacitor "plate". As most ham radio fans know, such a capacitor explodes when poled in the wrong way because of the electrochemical decomposition of the electrolyte. This is inconvenient. Replacing the electrolyte by a classical metal will not work, because a small scratch in the oxide layer will lead to a short. The liquid electrolyte has self-healing properties for the oxide layer. But the electrolyte can be replaced by polypyrrole! A large percentage of today's "electrolyte" capacitors are aluminium-aluminumoxide-polypyrrole capacitors.

\subsection{Through-hole plating}

Printed circuits are widely used in electronic appliances. To save space printed circuits are printed on both sides of a panel. To connect top and bottom a through-hole contact must be established. Of course, it is too expensive to drill a hole, stick a wire through and solder it on both sides. What one wants is to copper-plate the wall of the whole. For galvanic plating the substrate must be conducting (at least moderately conducting). Therefore it is pre-plated, conventionally by wet-chemical processes using hazardous solvents which are difficult to dispose. The novel process of polypyrrole pre-plating avoids the dangerous solvents and saves time in the total sequence of galvanic steps [8]. Today every second piece of consumer electronics, produced in Europe, contains polypyrrole.

\subsection{Batteries}

An electrochemical cell consists of two different metals in contact with an electrolyte. The metals need not be natural, they can well be synthetic, for example $p$-doped and $n$-doped polyacetylene, or lithium and polypyrrole. Since doping are redox processes, electrochemical doping and dedoping of conjugated polymers is charging and decharging of a battery. When some 15 years ago conjugated polymers were discovered to be "synthetic metals" there was great enthusiasm on polymer batteries: the weight difference between lead and plastic was striking. But although the polymer is light, the total battery is much less light because the dopant (e.g. $\mathrm{I}_{3}^{-}$) is heavy, and in addition it is not possible to store one charge unit per carbon atom. 
German (Varta), Japanese (Bridgestone-Saiko) and American (Allied Chemical) companies have successfully developed rechargeable polymer batteries (usually one electrode being a conventional metal, lithium). For a while these batteries were even on sale. They are about as good or as bad as nickel-cadmium-batteries. Since Ni/Cd batteries still sell quite well, there is no point in replacing them by something which is not better (but requires additional marketing expenses). The polymer battery is waiting on shelf till the existing batteries run into trouble, perhaps because of environmental problems with the heavy metal cadmium. A schematic diagram of an early-day polyacetylene battery is shown in Fig. 2.

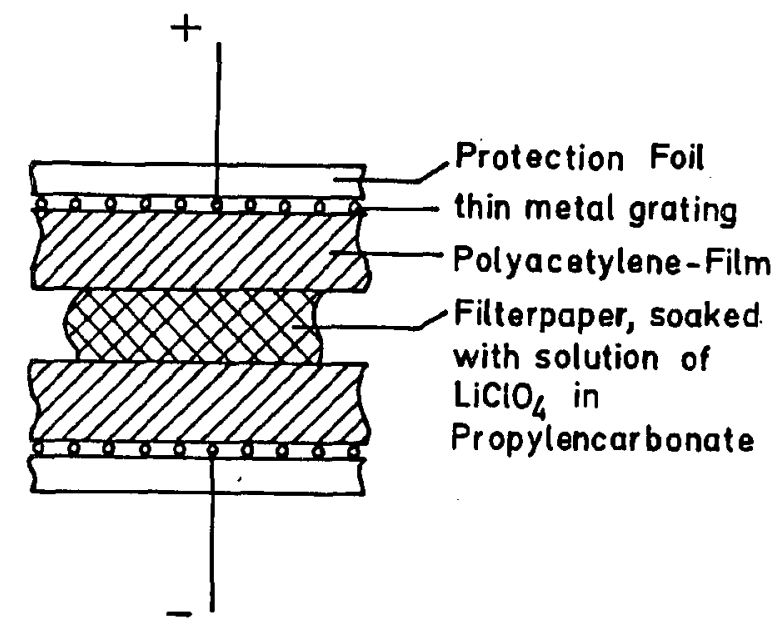

Fig. 2. Schematic view of an early-day polyacetylene battery.

\subsection{Solar cells}

A polymer solar cell is as simple as a polymer battery (see Fig. 3). Here not the metallic properties of heavily doped conjugated polymers are used but the semiconducting properties at moderate doping levels. In contact with a metal, a semiconductor usually forms a Schottky barrier. This happens with silicon and it also happens with many conjugated polymers. If light is shone into the Schottky barrier, an electric current can be drawn between the barrier contact and a back contact.

Speaking in terms of energy, silicon single crystals are fairly expensive. They are made by high temperature processes and a crystalline silicon solar cell has to run for about 8 years to collect the energy it has cost to produce the crystal. Polymers are energetically cheap. They are synthesized by catalytic chemical reactions at room temperature: why not spray a sequence of polymers layers onto a house roof and collect electricity from the gutter? After silicon people had learned to make solar cells from amorphous silicon and after amorphous silicon has become cheap, enthusiasm for polymer solar cells has rapidly decreased. From a certain price down, maintenance becomes more expensive than the investment and the cost of electricity produced by the solar cell is independent of the material price. 


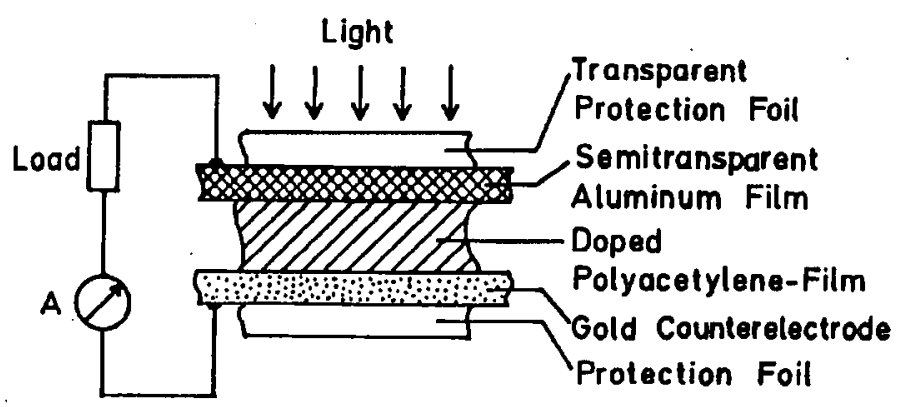

Fig. 3. Schematic view of a polymer solar cell.

\subsection{Loudspeakers, anti-noise-devices}

Figure 4 shows the schematic view of an electrostatic polymer loudspeaker [9]. A polymer membrane is suspended between two metal grids. The membrane (polyethylene, $6 \mu \mathrm{m}$ thick) is coated on both sides by a thin layer $(200 \mathrm{~nm})$ of polyaniline. High voltage is applied between the membrane and the grids and the high voltage is modulated by audio frequency. Thus the membrane moves with the audio frequency and the device acts as a loudspeaker. The essential feature is that this is a low-inertia loudspeaker which reacts immediately to the applied signal. Combined with a low-inertia pick-up such a system can be used as an anti-noise device, which emits anti-noise, $180^{\circ}$ phase shifted to the original noise and thus compensating it by destructive interference. The particular property of

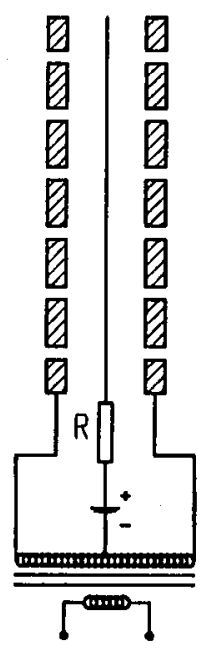

Fig. 4. Schematic view of a low-inertia electrostatic loudspeaker. 
the conjugated polymer used here is low area weight (for low inertia) and strong adhesiveness to the polyethylene membrane.

\subsection{Sensors}

Conjugated polymers have the reputation of being unstable. (Side note: not only conjugated polymers are unstable. Other polymers are unstable as well if they are pure, for industrial use various stabilizing additives have to be added.) Environmental sensitivity is the positive aspect of unstability. Therefore conjugated polymers can be used in sensors. Of course, there is the problem of selectivity and that of reversibility. There is a vast literature on sensorics and we content ourselves here with a Ref. [10].

\section{Miscellaneous application}

A survey of applications can never be comprehensive, in particular not, if the potentialities of future applications are also envisaged. In this section we just briefly summarize some applications which are less linked to the physical properties of conjugated double bonds (conductivity, energy gap, semiconducting aspects) but more to chemical and morphological aspects of the polymers.

\subsection{Actuators}

When conjugated polymers are doped they "swell": ions penetrate between the polymer chains and the sample expands. This expansion can be electrically controlled (electrochemical doping) and it can be used to generate mechanical motion in robotics ("artificial muscles") [11]. This has to be compared to piezoelectric actuators, where the motion is more precise, but where the stroke is much smaller.

\subsection{Anti-corrosion coating}

Because of the electrochemical properties some of the conjugated polymers can be used as protective layers to avoid corrosion. This has been proposed both for ship building and for dental implants.

\subsection{Drug release}

Doping and dedoping can be electrochemically controlled. During the redox process ions migrate into or out of the polymers. Some of these ions can be fairly complicated, in particular they can be organic ions onto which pharmaceutically active species can be attached. This can be applied in medicine to electrically controlled drug release.

\subsection{Biocompatible wires}

Most inorganic materials are ejected from the human body after some time. Therefore it is impractical to pass platinum wires through the human skin in the same way as we have feed-throughs into vacuum chambers. Some conjugated polymers have a better biocompatibility and might be used, for example, to activate muscles by electrical pulses. 


\subsection{Monodisperse particles}

Under certain conditions polypyrrole has the tendency to form vesicles (mini-bubbles) of well-defined size. Such dispersions are useful in several biological applications, in particular because chemistry allows to attach further agents on the outer surfaces of the vesicles [12].

\subsection{Gas separation membranes}

Films of conjugated polymers usually have a very complex morphology: well ordered crystalline microdomains are separated by disordered regions. This leads to problems if one wants to calculate transport properties such as the electrical conductivity. But the disordered regions allow gases to pass the film, often in a selective way. Thus conjugated polymers can form gas separation membranes [13].

\section{Large scale electronics}

Silicon technology has proven to be superior for device miniaturization not only in microelectronics but also in micromechanics. But will silicon also be the material of choice if large area devices are needed? We have already encountered one case of large area devices: solar cells (Sec. 2.8). Another example would be large conducting and transparent electrodes [14]. State of the art is ITO sputtered onto quartz (ITO = indium tin oxide). Alternatives with conjugated polymers are thin layers of polypyrrole or polyaniline.

The "hot issue" in this field certainly is organic light emitting devices (LED's) for large, flat, and flexible video screens. Present television sets use cathode ray tubes. These Braunian tubes are the last left-over of vacuum electronics and replacement by solid state technology is highly desirable. Liquid crystal displays is one approach, but liquid crystals are not shining themselves, they act as filters for illumination from behind. Not so semiconducting light emitting diodes. Diodes made from our star semiconductor silicon do not luminesce. Therefore today semiconductor LED's are made from III-V semiconductors (gallium arsenide) and large diode arrays (of several square meters) would be prohibitively expensive. Porous or surface-modified silicon might open this field to silicon technology in the future, but presently the technological problem is unsolved. Consequently organic materials might have a chance, and indeed, much $R \& D$ effort has arisen in Europe, Japan and in the US, after the first scientific publications [15-18].

Figure 5 illustrates the physics of a light emitting device: an (organic or inorganic) semiconductor is sandwiched between two metal electrodes. Applying voltage to the electrodes the Fermi levels are adjusted in such a way that one electrode can inject electrons into the conduction band $\left(\pi^{*}\right.$-band) and the other holes into the valence band ( $\pi$-band) (note: hole injection is electron withdrawal). If electron and hole recombine radiatively, the device emits light. The better the work functions of the electrode metals are adjusted to the valence and conduction bands, the less voltage has to be applied to operate the device. The colour of the emitted light depends on the $\pi-\pi^{*}$ gap and by choosing appropriate polymers and by intelligent molecular engineering any colours in the visible range can be obtained. A schematic view of a polymer LED is shown in Fig. 6 [19]. 


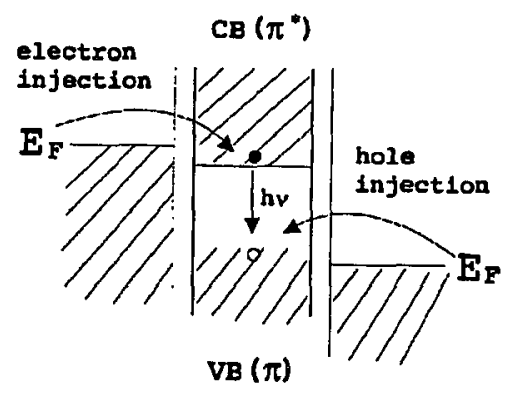

Fig. 5. Physics of LED.

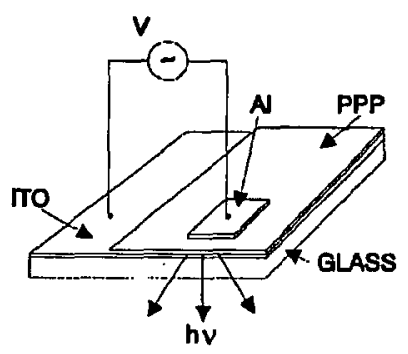

Fig. 6. Schematic view of a polymer LED.

Today the luminosity and the lifetime of organic LED's is not yet sufficient for technical applications, but if $R \& D$ efforts continue, commercialization can be expected for the next couple of years.

\section{Molecular electronics}

Figure 7 show the continuous progress of silicon miniaturization. The lateral dimension of a computing element is plotted versus the year (AD). Such plots are known as Morre's plot and were originally used to demonstrate the US\$ price per bit in a VLSI memory. It turns out that lateral dimension (and price) decrease exponentially. If there is no saturation, in 2030 we would reach $1 \AA$ and a field effect transistor would consist of 1 atom only. Certainly there will be practical and theoretical limits before that. Will the use of organic materials instead of silicon help in miniaturization? Because organic chemistry is richer than silicon chemistry? Because in organics we have stronger electron lattice coupling (we can couple to conformational changes and thus we can better localize excitations)? Probably it will take us several years or even decades to treat these questions.

The term "molecular electronics" is not well defined. It is used both for "electronics on a molecular level" and for "molecular materials for electronics" [20]. Some relevant conference proceedings are cited in Refs. [21-24].

A key issue in electronics on a molecular level are switching molecules. Figure 8 shows bianthrone as one example out of several, where molecules exist in two 


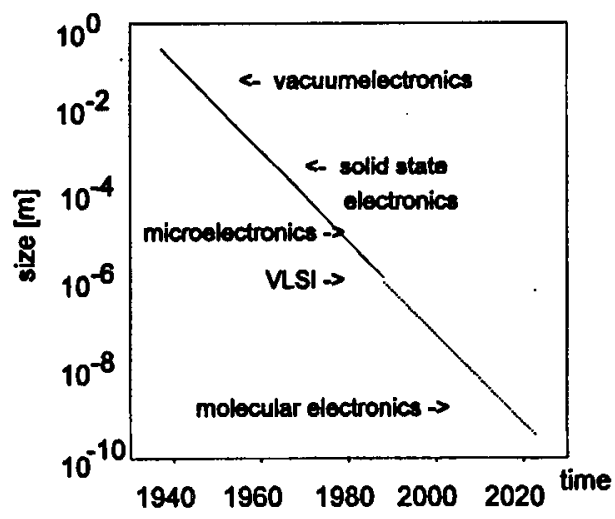

Fig. 7. Continuous progress in silicon device miniaturization.

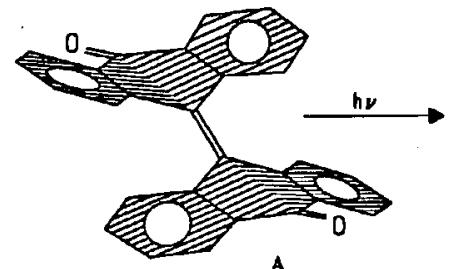

A

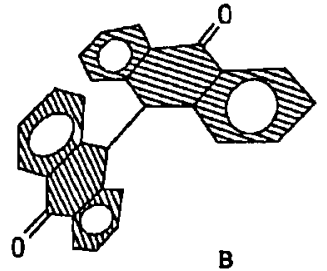

B

Fig. 8. Bianthrone shown in two conformations; (A) the stable puckered form, (B) two twisted planar half molecules.

forms and where one form switches into the other by absorption of light. This particular molecule illustrates nicely the conformational changes involved. In form $B$ the anthracene units are planar but they are twisted with respect to each other. In form $A$ there is no twist but the anthracene units are puckered. Typical matters of research are: how quickly do the molecules shift, how selective is the switching, what are the rates for spontaneous backswitching - how can these parameters be modified by adding functional groups at various positions [25]. The next generation of questions would be: how can we connect molecular switches, how would they interact, how can we use these interactions for logic operations? It is easy to imagine that there is still a long way to go till we have a molecular computer!

Certainly we can build holographic computers, where the hologram is stored in an organic film containing molecular switches. An example are membranes of bacteriorhodopsine [26], which, in a certain way, is related to conjugated polymers - at least rhodopsine contains strands with conjugated double bonds. But even a polyacetylene-based holographic computer has been built [27]. By spectral hole burning we have "access to a further dimension" in hologram storing and can largely increase the storage capacity (up to 2000 holograms simultane- 


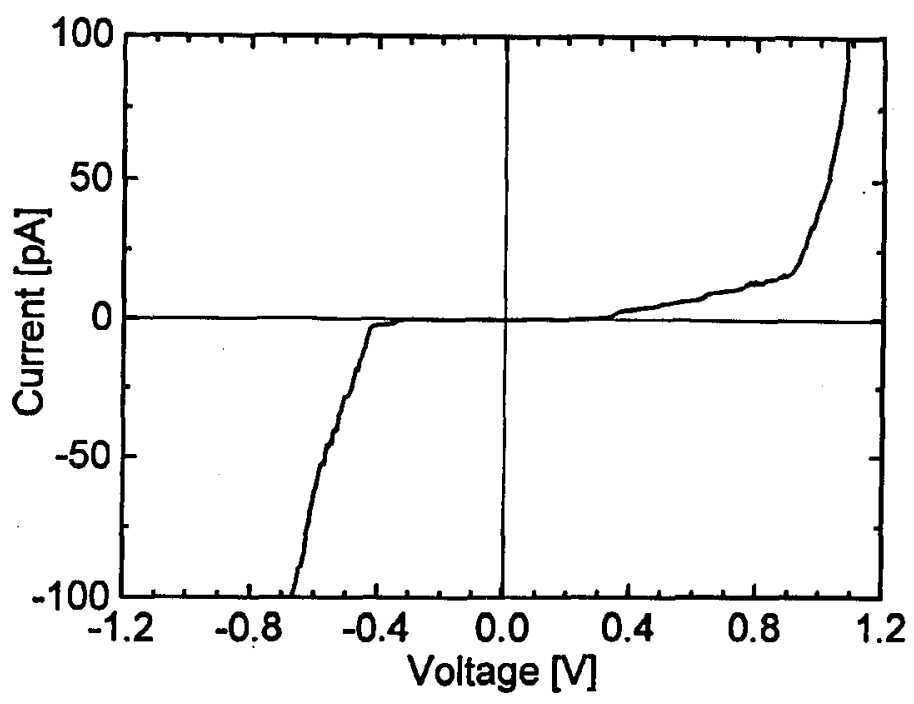

Fig. 9. Molecular rectification and single electron effects in organic films.

ously) [28]. One problem in "molecular materials for electronics" is that of organic rectifiers or even molecular rectifiers [29]. An example of such studies is shown in Fig. 9 [30]. This is an attempt to build the organic analogue of GaAs/AlAs heterostructures. The organic heterostructure involves Langmuir-Blodgett films of metallophthalocyanine and perylenetetracarboxyldiimide derivatives. Both substances are organic semiconductors based on conjugated macrocycles. As GaAs and AlAs they have different band gaps ( $\pi-\pi^{*}$ separation). The figure displays the current voltage characteristic (at liquid helium temperature) of a sandwich gold6 layers metallocyanine-6 layers perylenetetracarboxyldiimide-gold. The sandwich is blocking up to a certain threshold voltage, but the threshold is asymmetric, 0.4 and $0.8 \mathrm{~V}$, respectively. If operated between 0.4 and $0.8 \mathrm{~V}$ the sandwich is rectifying. In addition to rectifying behaviour, characteristic steps are observed. These are tentatively interpreted as single electron Coulomb blockades in analogy to single electron effects in inorganic quantum dots. The organic heterolayers discussed here are not intended for immediate industrial applications. The goal is rather to elaborate the physical and chemical basis for a future evaluation of application potentialities of electroactive organic materials in electronics.

\section{Acknowledgment}

This work has emerged from the Commission of the European Communities BRITE/EURAM Research Project HICOPOL (Highly Oriented Highly Conducting Polymers) and ESPRIT Network of Excellence NEOME (New Electroactive Organic Materials for Electronics). 


\section{References}

[1] H.J. Queisser, Kristallene Krisen, R. Piper GmbH\&Co.KG, München 1985.

[2] For review articles on conjugated polymers see e.g.: S. Roth, H. Bleier, Advances in Physics 36, 385 (1987); Handbook of Conducting Polymers, Vol. 1 and Vol. 2, Ed. T.A. Skotheim, Marcel Dekker, New York 1986; Faraday Discussions of the Chemical Society, No. 88, Charge Transfer in Polymeric Systems, The Royal Society of Chemistry, London 1989.

[3] A.G. MacDiarmid, private communication.

[4] L. Belleville, Laser Focus World, October 1989, p. 69.

[5] J. Hocker, F. Jonas, H.-K. Müller, B. Broich, in: Elektrisch leitende Kunststoffe, 1st ed., Eds. H.J. Mair, S. Roth, Carl Hanser Verlag, München 1986, p. 181.

[6] M. Angelopoulos, J.M. Shaw, J.J. Ritsko, in: Science and Applications of Conducting Polymers, Eds. W.R. Salaneck, D.T. Clark, E.J. Samuelsen, Adam Hilger, Bristol 1991, p. 63.

[7] U. Leute, in: Elektrisch leitende Kunststoffe, 2nd ed., Eds. H.J. Mair, S. Roth, Carl Hanser Verlag, München 1989, p. 521.

[8] W. Metzger, J. Hupe, W. Kronenberg, Plating Surf. Finishing 28 (1990).

[9] B. Wessling, Fa. Zipperling, private communication.

[10] Sensors - A Comprehensive Survey, Eds. W. Göpel, J. Hesse, N. Zemel, VCH, Weinheim 1991.

[11] R.H. Baughman, L.W. Shacklette, in: Science and Applications of Conducting Polymers, Eds. W.R. Salaneck, D.T. Clark, E.J. Samuelsen, Adam Hilger, Bristol 1991, p. 47; T.F. Otero, J. Rodriguez, in: Intrinsically. Conducting Polymers: An Emerging Technology, Ed. M. Aldissi, Kluwer Academic Publishers, Dordrecht 1993, p. 179.

[12] S.P. Armes, in: Intrinsically Conducting Polymers: An Emerging Technology, Ed. M. Aldissi, Kluwer Academic Publishers, Dordrecht 1993, p. 35.

[13] M.R. Anderson, B.R. Mattes, H. Reiss, R.B. Kaner, Synth. Met. 41, 1151 (1991).

[14] R.J. Visser, lecture at NEOME workshop in E-MRS Symposium: Open Forum on the Role of Industry in the Development of New Materials for New Devices, Strasbourg 1993, unpublished (NEOME = Network of Excellence on New Electroactive Organic Materials for Electronics).

[15] J.H. Burroughes, D.D.C. Bradley, A.R. Brown, R.N. Marks, K. Mackay, R.H. Friend, P.L. Burns, A.B. Holmes, Nature 347, 539 (1990).

[16] Y. Ohmori, M. Uchida, K. Muro, K. Yoshino, Jpn. J. Appl. Phys. 30, L1938 (1991).

[17] G. Grem, G. Leditzky, B. Ullrich, G. Leising, Adv. Mater. 4, 36 (1992).

[18] G. Gustafsson, Y. Cao, G.M. Treacy, F. Kavetter, N. Colaneri, A.J. Heeger, Nature 357, 477 (1992).

[19] J. Stampfl, W. Tritthart, G. Leising, NEOME Report No.4: Optics Report, unpublished, 1994 (NEOME = Network of Excellence on New Electroactive Organic Materials for Electronics).

[20] D. Bloor, M. Hanack, A. Le Méhauté, R. Lazzaroni, J.P. Rabe, S. Roth, H. Sasabe, in: Conjugated Polymeric Materials, Opportunities in Electronics, Optoelectronics, and Molecular Electronics, Eds. J.L. Bredas, R.R. Chance, NATO ASI Series, Series E: Applied Sciences, Vol. 182, Kluwer Academic Publishers, Dordrecht 1990, p. 587 . 
[21] Molecular Electronics - Biosensor and Biocomputers, Ed. F.T. Hong, Plenum Press, New York 1989.

[22] Molecular Electronics - Science and Technology, Ed. A. Aviram, United Engineering Trustees, New York 1989.

[23] Synthetic Metals for Non-Linear Optics and Electronics (Proceedings of Symposium $E$ of the 1992 E-MRS Spring Conference), Eds. C. Taliani, Z.V. Vardeny, Y. Maruyama, North-Holland, Amsterdam 1993.

[24] Molecular Electronics (Proceedings of Symposium H of the 1993 E-MRS Spring Conference), Eds. Ch. Ziegler, W. Göpel, G. Zerbi, North-Holland, Amsterdam 1993.

[25] K. Schaumburg, J.-M. Lehn, V. Goulle, S. Roth, H. Byrne, S. Hagen, J. Poplawski, K. Brunfeldt, K. Bechgaard, T. Bjornholm, P. Frederiksen, M. Jorgensen, K. Lerstrup, P. Sommer-Larsen, O. Goscinski, J.-L. Calais, L. Eriksson, in: Nanostructures Based on Molecular Materials, Eds. W. Göpel, Ch. Ziegler, VCH, Weinheim 1992, p. 153.

[26] C. Bräuchle, N. Hampp, D. Oesterhelt, Adv. Mater. 3, 420 (1991).

[27] D.P. Foote, in: ME-News No. 13, 1992, Ed. M. Petty, Center for Molecular Electronics, Durham (UK) 1992.

[28] B. Kohler, S. Bennet, A. Renn, U.P. Wild, Opt. Lett. 18, 2144 (1993).

[29] R.M. Metzger, in: Electricity and Magnetism in Biology and Medicine, Ed. M. Blank, San Francisco Press Inc., San Francisco 1993.

[30] C.M. Fischer, M. Burghard, S. Roth, K. von Klitzing, Europhys. Lett. 28, 129 (1994). 